\title{
Electromagnetic mode profile shaping in waveguides
}

\author{
Jonathan Gratus $^{1,2} \cdot$ Paul Kinsler ${ }^{1,2} \cdot$ Rosa Letizia $^{2,3} \cdot$ Taylor Boyd $^{1,2}$
}

Received: 5 October 2016/ Accepted: 3 December 2016/Published online: 3 January 2017

(C) The Author(s) 2017. This article is published with open access at Springerlink.com

\begin{abstract}
Electromagnetic mode profile shaping would be a very useful technique, with applications including in accelerator science and data transmission. Two methods are proposed: one varying the permittivity and the other using a wire medium. By the use of Mathieu functions, we demonstrate a flatter, a triangular and a peakier profile. The first two may be used to manipulate longer particle bunches, while the last could be used on shorter bunches and in data transmission. It is also demonstrated how further improvements can be achieved by going beyond Mathieu functions.
\end{abstract}

\section{Introduction}

Electromagnetic modes in a waveguide with uniform cross section have a sinusoidal profile. There are many situations where non-sinusoidal waves may be particularly useful. In accelerator science, for example, one may wish to have a flatter profile. The waveform in Fig. 1 would accelerate a

Jonathan Gratus

j.gratus@lancaster.ac.uk

Paul Kinsler

dr.paul.kinsler@physics.org

Rosa Letizia

r.letizia@lancaster.ac.uk

Taylor Boyd

w.boyd@lancaster.ac.uk

1 Physics Department, Lancaster University, Lancaster LA1 4YB, UK

2 Cockcroft Institute, Daresbury, Warrington WA4 4AD, UK

3 Engineering Department, Lancaster University, Lancaster LA1 4YW, UK longer bunch, and therefore more electrons, for a given peak power. By contrast a peakier profile, Fig. 2, would accelerate shorter bunches for the same total power. In addition due to the steeper gradient, it would enable one to give a shorter bunch more chirp which is necessary for bunch compression. The peakier profiles would also be useful for data transmission where one can have the higher peak when the signal is being observed. Intermediate between the flatter and peakier profile is a triangular profile as given in Fig 3. This has the advantage of having a significant part of the profile having a constant slope. In terms of interactions with electron bunches, this would give longer bunches a sustained chirp. Figures 1,2 and 3 are all examples of waveforms based on Mathieu functions, which often have convenient properties. However, we can go beyond Mathieu functions. In Figs. 4 and 5, we demonstrate how an even flatter profile can be achieved.

In this article we modify the mode profile by modifying the constitutive relations, in particular the permittivity. To simplify the analysis, we consider the permittivity $\epsilon$ to be periodic in $z$ the direction of propagation.

We consider two scenarios. The first is for transverse waves, Sect. 2. In this case, we choose a permittivity which depends on frequency and position [2]. The use of varying the permittivity combined with the use of Mathieu functions was considered by El Haddad [6], where the transmission functions were calculated. However, the author did not consider the use of this to modify the field profile. There are many methods for making such a medium. For example, one can compose the medium from many thin slices of media, each with a different value of $\epsilon$, thereby approximating the continuous variation. Another method is to set up a standing wave in a nonlinear material. The linearised permittivity about this solution would have the spatial dependence as desired. However, for the examples 


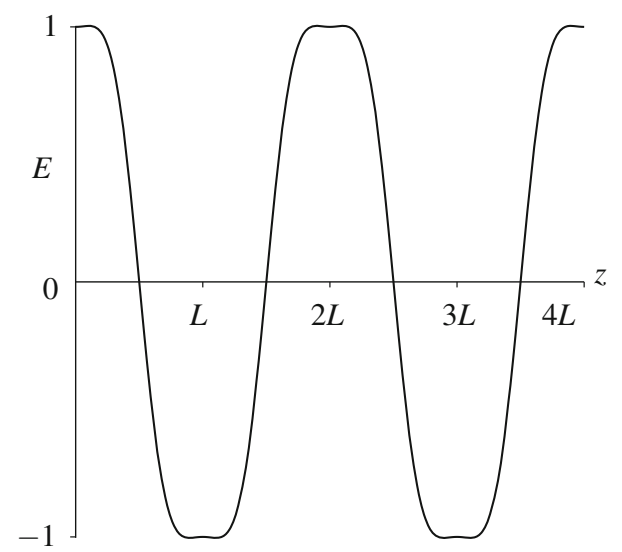

Fig. 1 A flatter solution to Mathieu's equation: In (3) with $q=0.8$ and $a=A_{1}(0.8)=1.711$, where $A_{n}(q)$ is the Mathieu-A function

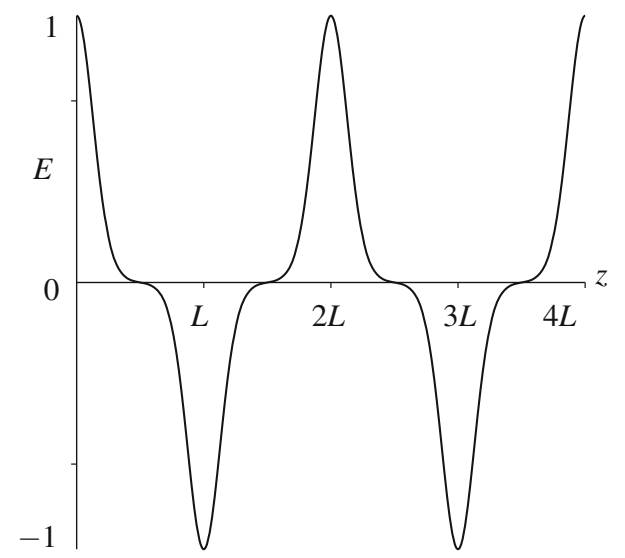

Fig. 2 A peakier solution to Mathieu's equation: In (3) with $q=$ -10.0 and $a=A_{1}(-10.0)=-13.937$

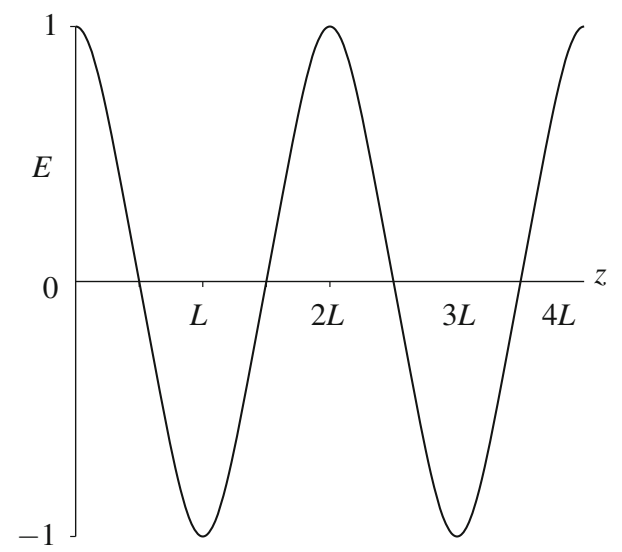

Fig. 3 A triangular solution to Mathieu's equation which has the advantage of having a significant part of the profile having a constant slope: In (3) with $q=-0.329$ and $a=A_{1}(-0.329)=0.658$

in Figs. 1 and 4 given in this article to work would require regions to have negative permittivity, $\epsilon<0$ which would

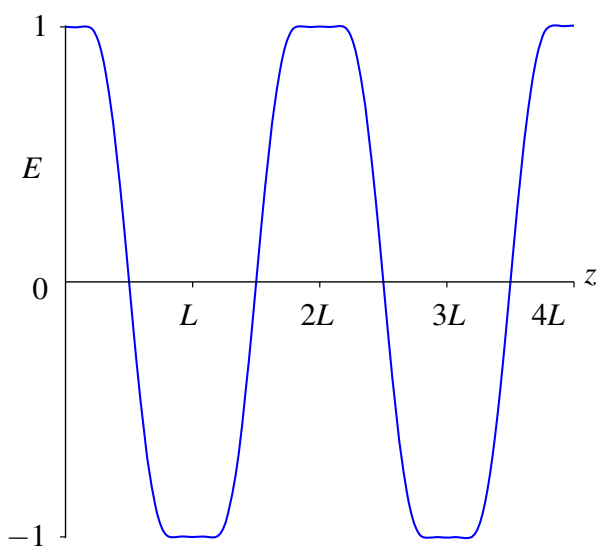

Fig. 4 An even flatter profile: $A$ solution to the equation $\pi^{2} L^{-2} E^{\prime \prime}+(1.9266-2.2 \cos (2 \pi Z / L)+0.4 \cos (8 \pi Z / L)) E=0$

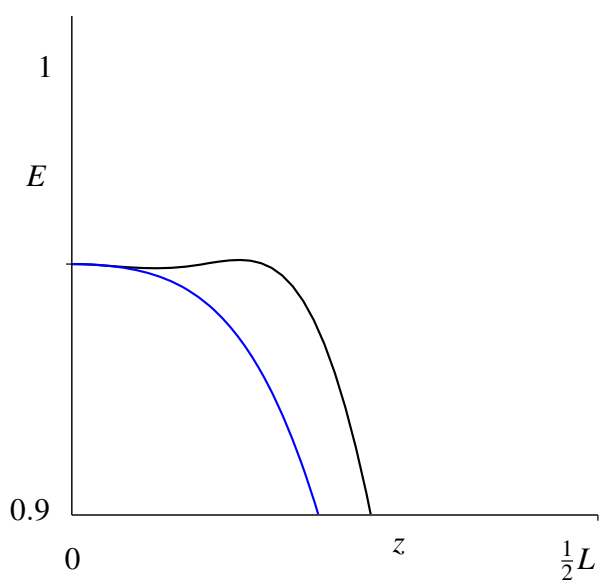

Fig. 5 Close up showing difference between the profiles in Figs. 1 and 4

require some kind of metamaterial [3, 14-17, 19]. It also does not work for longitudinal waves. An alternative to prescribing the permittivity profile and asking if the resulting electric field profile is desirable is to start with a particular field profile and attempt to see whether it can be constructed with a realistic permittivity. This is investigated in subsequent work [7].

A second scenario, Sect. 3, is to make $\epsilon$ spatially dispersive as well as inhomogeneous. That is, we set $\epsilon=$ $\epsilon(\omega, k, z)$ where $k$ is the Fourier conjugate variable associated with $z$. It is natural to ask the meaning of a function depending on both $k$ and $z$. This is addressed in $[2,8,10,11]$. The method, as described below, is to replace the permittivity function with a differential equation. A wire medium $[1,4,9,22,23]$ is naturally spatially dispersive, and by varying the radius of the wires periodically in $z$ will naturally give it the correct spatial inhomogeneity (Fig. 12). 


\section{Transverse modes periodic inhomogeneous permittivity}

Consider the single-frequency transverse mode with $\mathbf{E}=e^{i \omega t} \tilde{E}(z) \mathbf{i}, \mathbf{P}=e^{i \omega t} \tilde{P}(z) \mathbf{i}$ and $\mathbf{H}=e^{i \omega t} \tilde{H}(z) \mathbf{j}$, together with the permittivity $\epsilon(\omega, z)=\epsilon_{0} \epsilon_{r}(\omega, z)$ and vacuum permeability $\mu_{0}$. Here $\{\mathbf{i}, \mathbf{j}, \mathbf{k}\}$ are the unit vectors along $\{x, y, z\}$. Then Maxwell's equations give

$\tilde{E}^{\prime \prime}+\omega^{2} c^{-2} \epsilon_{r}(\omega, z) \tilde{E}=0$

where $\iota=\frac{d}{d z}$. Set

$\epsilon_{r}(\omega, z)=\pi^{2} c^{2} \omega^{-2} L^{-2}(a(\omega)-2 q(\omega) \cos (2 \pi z / L))$

where $L$ is the period of the $\epsilon$ variation: $\epsilon(\omega, z)=\epsilon(\omega, z+L)$. Then we get the (rescaled) Mathieu's equations

$\pi^{-2} L^{2} \tilde{E}^{\prime \prime}+(a-2 q \cos (2 \pi z / L)) \tilde{E}=0$

It is easy to model spatially varying permittivity numerically using the CST package [5], when $\epsilon>0$. Since $a=$ $A_{1}(q)$ where $A_{n}(q)$ is the Mathieu-A function, then from (2) we require that $A_{1}(q)>|q|$. This implies that

$-0.329<q<0.889$

Conveniently, the two limits of this range correspond to the triangular and flatter profiles. To validate these results, numerical simulations of these profiles have been performed using 20 slabs of constant $\epsilon_{r}$ by the eigen mode solver of CST Microwave Studio [5]. Periodic, PEC and PMC boundary conditions are chosen along the $z, x$ and $y$ directions, respectively. Figures. 6 and 7 show the comparison between the $E$-field profile for the modelled and simulated modes, where a very good agreement can be observed. The simulated $E$-field profile on the $x-y$ plane is given in Figs. 8 and 9 for the flatter and triangular modes, respectively.

These were implemented directly into CST, by using 20 slabs of constant $\epsilon_{r}$. To confirm these modes, we have run a CST simulation as shown in Figs. 6, 7, 8 and 9.

If we are allowed to have negative $\epsilon_{r}$, then one can create more extreme shapes. For example, in Figs. 4 and 5 we add a higher harmonic to produce an even flatter profile, whereas in Fig. 2 we obtain a much peakier solution. One must be careful, however, with simulating these. We have used MPB [13] to simulate the fields for $q=0.8$ to $q=1$, Fig. 10. We see that the region with negative $\epsilon_{r}$ has numerical instabilities.

The oscillation of the permittivity at the order of the wavelength implies that the structure is similar to a onedimensional photonic crystal or Bragg mirror $[12,18,20,21,24]$. One can ask what the transmission

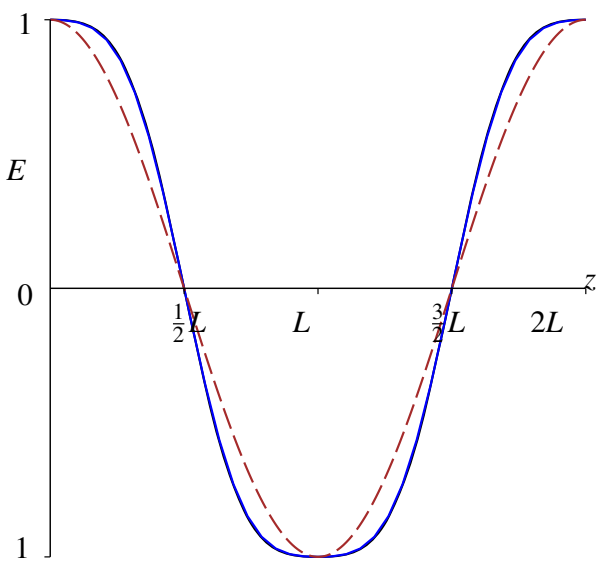

Fig. 6 Comparison of the simulated $E_{x}$-field (blue) [Fig. 8] for a flatter profile, as in Fig. 1, with the predicted curve (black). However, the two curves are so close that one cannot distinguish them in this figure. In comparison with the cosine (dashed brown)

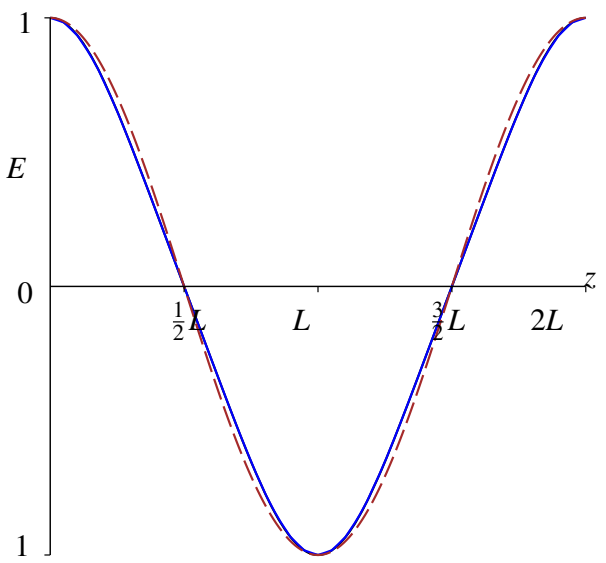

Fig. 7 Comparison of the simulated $E_{x}$-field (blue) [Fig. 9] for a triangular profile, as in Fig. 3, with the predicted curve (black). Likewise, the two curves are almost identical. In comparison with the cosine (dashed brown)

coefficient is for waves coming into this medium from a vacuum. In Fig. 11 we use CST to calculate the transmission coefficients for the flatter profile as in Fig. 8. We see that at the proposed frequency of $2.9 \mathrm{GHz}$ there is a high transmission and we are at the edge of a band gap.

\section{Wire media: longitudinal modes with spatially dispersive inhomogeneous permittivity}

Consider longitudinal modes so that the electric and polarisation fields are longitudinal and the magnetic field vanishes, i.e. $\mathbf{E}=e^{i \omega t} \tilde{E}(z) \mathbf{k}, \mathbf{P}=e^{i \omega t} \tilde{P}(z) \mathbf{k}$ and $\mathbf{B}=\mathbf{0}$, then Maxwell's equations are automatically satisfied if 


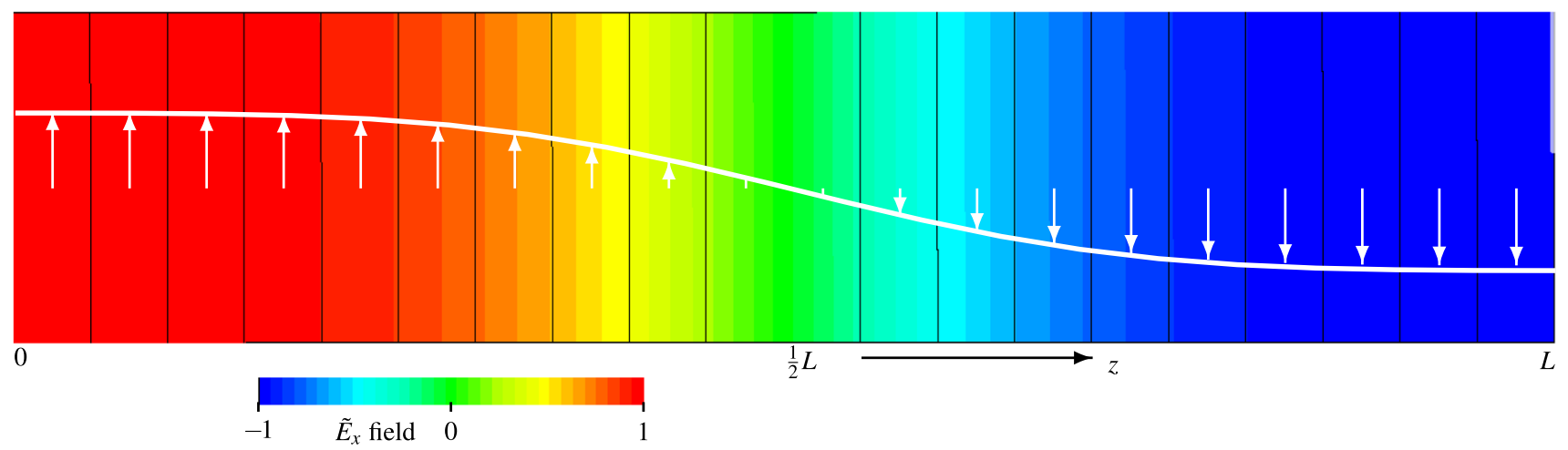

Fig. 8 CST simulated plot of $E_{x}$ field for the flatter profile, as in Fig. 1

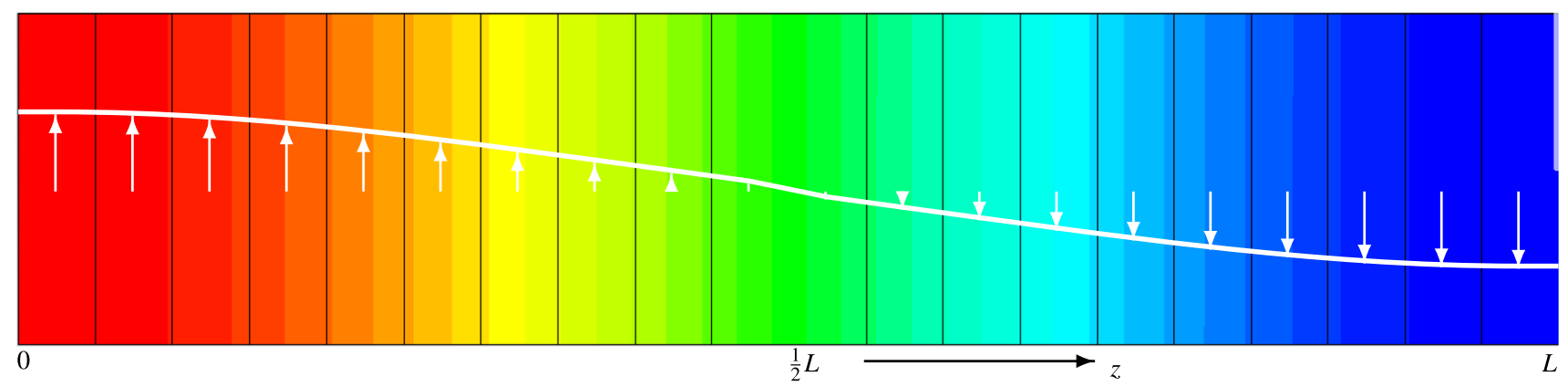

Fig. 9 CST simulated plot of $E_{x}$ field for the triangular profile, as in Fig. 3

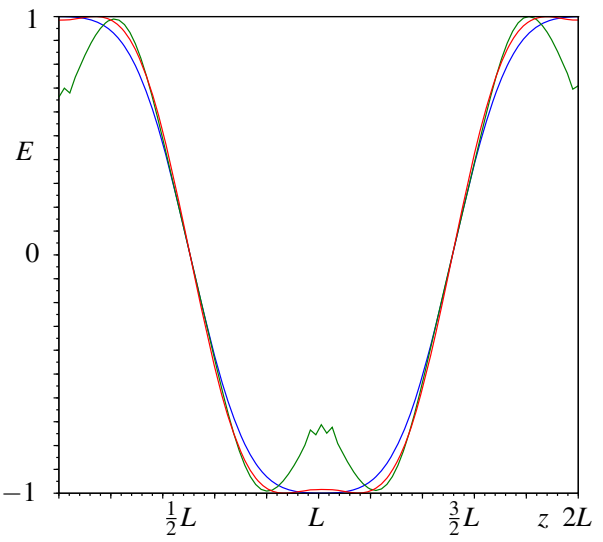

Fig. 10 MPB calculation of the flatter modes with $q=0.8$ (blue), $q=0.9$ (green) and $q=1.0$ (red). The green curve shows numerical instability

$$
\epsilon_{0} \tilde{E}+\tilde{P}=0
$$

i.e. $\mathbf{D}=\mathbf{0}$, thus we are looking for epsilon near zero (ENZ) media. When the medium is homogeneous, we will use an empirical model of the permittivity via

$\epsilon(\omega, k)=\epsilon_{0}-\frac{\epsilon_{0} k_{p}^{2}}{\mathcal{L}(\omega)-\beta^{2} k^{2}}$

Combining (5) and (6), we obtain the dispersion relation

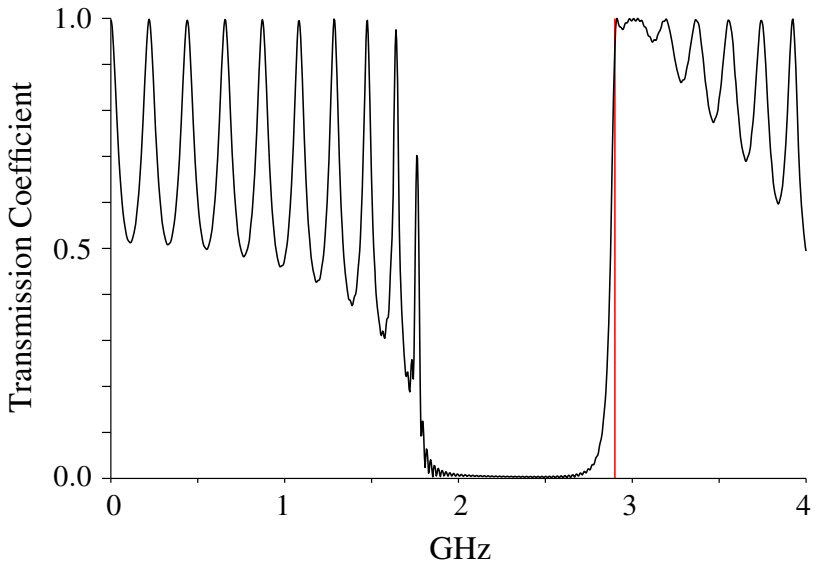

Fig. 11 Transmission coefficients for a periodically varying permittivity as in Fig. 8. This is implemented in CST using 10 cells. The transmission is very high and corresponds to the right edge of the band gap at around $2.89 \mathrm{GHz}($ red $)$

$$
\mathcal{L}(\omega)-\beta^{2} k^{2}=k_{p}^{2}
$$

Taking the Fourier transform of $0=\epsilon_{0} \hat{E}(k)+\hat{P}(k)=$ $\epsilon(\omega, k) \hat{E}(k)$ with respect to $k$ using $\tilde{P}(z)=\int_{-\infty}^{\infty} e^{i k z} \hat{P}(k) d k$, one obtains the differential equation

$\beta^{2} \tilde{E}^{\prime \prime}+\mathcal{L}(\omega) \tilde{E}=k_{p}^{2} \tilde{E}$ 


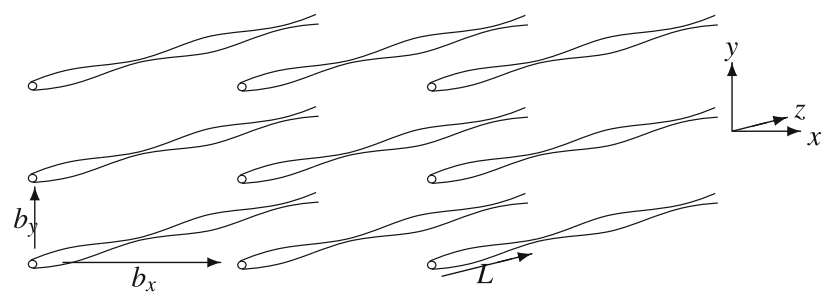

Fig. 12 Wire medium with a periodic variation in the radius of the wires. The inter wire spacing is $\left(b_{x}, b_{y}\right)$ and the period of the longitudinal variation is $L$

The simplest method to include an inhomogeneity in the permittivity is to let the plasma frequency $k_{p}$ depend on position $z$, that is $k_{p}=k_{p}(z)$. Thus (8) becomes

$\beta^{2} \tilde{E}^{\prime \prime}+\left(\mathcal{L}(\omega)+k_{p}(z)^{2}\right) \tilde{E}=0$

Again by choosing the appropriate periodic function for $k_{p}(z)$, one can replace (9) by the Mathieu equation.

As stated in the introduction, this can be created with a wire medium, as described in more detail in [9].

\section{Conclusion}

Two methods of mode profile shaping are suggested. One using transverse waves and a varying permittivity. The second is by using a wire medium with periodic variation in the radius of the wire. In the first method, we observed that for the more extreme profiles such as the peakier profile, Fig. 2, and the flatter profile Fig. 4 require $\epsilon<0$. This is challenging for the numerical solvers CST and MPB. In addition, one would suspect it would also be challenging to implement experimentally. Since we have implemented a 1-dimensional photonic crystal, we see from Fig.11 that there are band gaps and the operating frequency is at the edge of this band gap. Fortunately the transmission is high.

We are currently implementing numerically the wire medium to optimise the shape of the wires to form the desired profile shape.

Acknowledgements The authors are grateful for the support provided by STFC (the Cockcroft Institute ST/G008248/1) and EPSRC (the Alpha-X project EP/J018171/1 and EP/N028694/1).

Open Access This article is distributed under the terms of the Creative Commons Attribution 4.0 International License (http://crea tivecommons.org/licenses/by/4.0/), which permits unrestricted use, distribution, and reproduction in any medium, provided you give appropriate credit to the original author(s) and the source, provide a link to the Creative Commons license, and indicate if changes were made.

\section{References}

1. P. Belov, R. Marques, S. Maslovski, I. Nefedov, M. Silveirinha, C. Simovski, S. Tretyakov, Strong spatial dispersion in wire media in the very large wavelength limit. Phys. Rev. B 67(11), 113103 (2003)

2. T. Boyd, P. Kinsler, J. Gratus, R. Letizia, Electromagnetic mode profile shaping in waveguides, pp. 725-727. IEEE (2016) doi:10. 1109/URSI-EMTS.2016.7571502

3. F. Capolino, Theory and Phenomena of Metamaterials (CRC Press, Boca Raton, 2009)

4. J.T. Costa, M.G. Silveirinha, Macroscopic electromagnetic response of arbitrarily shaped spatially dispersive bodies formed by metallic wires. Phys. Rev. B 86(7), 075129 (2012)

5. CST STUDIO SUITE. [Online].: CST AG, Germany. http:// www.cst.com

6. A. El Haddad, Exact analytical solution for the electromagnetic wave propagation in a photonic band gaps material with sinusoidal periodicity of dielectric permittivity. Optik-Int. J. Light Electron Opt. 127(4), 1627-1629 (2016)

7. J. Gratus, P. Kinsler, R. Letizia, T. Boyd, Customised mode profiles using functional materials. Phys. Rev. A (In review) arxiv: 1607.04313 (2016)

8. J. Gratus, M. McCormack, Spatially dispersive inhomogeneous electromagnetic media with periodic structure. J. Opt. 17(2), 025105 (2015)

9. J. Gratus, M. McCormack, R. Letizia, Spatially dispersive inhomogeneous dielectric wire media with periodic structure. In: Proceedings of PIERS 2015 in Prague, July 6-9, 2015, pp. 675-680. The Electromagnetics Academy (2015)

10. J. Gratus, R.W. Tucker, Covariant constitutive relations, Landau damping and non-stationary inhomogeneous plasmas. Prog. Elec. Res. M 13, 145-156 (2010)

11. J. Gratus, R.W. Tucker, Covariant constitutive relations and relativistic inhomogeneous plasmas. J. Math. Phys. 52, 042901 (2011)

12. J.D. Joannopoulos, S.G. Johnson, J.N. Winn, R.D. Meade, Photonic Crystals: Molding the Flow of Light (Princeton University Press, Princeton, 2011)

13. S.G. Johnson, and the Joannopoulos Ab Initio Physics Group MIT: MIT photonic bands. http://ab-initio.mit.edu/wiki/index. php/MIT_Photonic_Bands

14. S.E. Lauro, A. Toscano, L. Vegni, Symmetrical coupled microstrip lines with epsilon negative metamaterial loading. IEEE Trans. Magn. 45(3), 1182-1185 (2009)

15. L. Mandelshtam, Complete Works, vol. 5 (Academy of Sciences, USSR, 1950)

16. M. Notomi, Theory of light propagation in strongly modulated photonic crystals: refractionlike behavior in the vicinity of the photonic band gap. Phys. Rev. B 62(16), 10696 (2000)

17. G. Oliveri, D.H. Werner, A. Massa, Reconfigurable electromagnetics through metamaterials-a review. Proc. IEEE 103(7), 1034-1056 (2015)

18. L. Pilozzi, A. D'Andrea, K. Cho, Spatial dispersion effects on the optical properties of a resonant Bragg reflector. Phys. Rev. B 69(20), 205311 (2004)

19. T. Rowe, J.H. Booske, N. Behdad, Metamaterial-enhanced resistive wall amplifiers: theory and particle-in-cell simulations. IEEE Trans. Plasma Sci. 43(7), 2123-2131 (2015)

20. H.E. Ruda, J.C. Polanyi, J.S. Yang, Z. Wu, U. Philipose, T. Xu, S. Yang, K. Kavanagh, J. Liu, L. Yang et al., Developing 1d nanostructure arrays for future nanophotonics. Nanoscale Res. Lett. 1(2), 99-119 (2006) 
21. J.D. Ryckman, S.M. Weiss, Localized field enhancements in guided and defect modes of a periodic slot waveguide. IEEE Photonics J. 3(6), 986-995 (2011)

22. M.G. Silveirinha, Additional boundary condition for the wire medium. IEEE Trans. Antennas Propag. 54(6), 1766-1780 (2006)

23. W. Song, Z. Yang, X.Q. Sheng, Y. Hao, Accurate modeling of high order spatial dispersion of wire medium. Opt. Express 21(24), 29836-29846 (2013)
24. P. Xie, Z. Zhang, K. Wong, Simultaneous high-efficiency secondand third-harmonic generation in a 1-d semiconductor photonic crystal. IEEE J. Quantum Electron. 43(9), 804-809 (2007) 